\title{
Native 1st Metatarso-Phalangeal Joint Infection: A Rare Case Report
}

\author{
Efthymios lliopoulos, Natasha Hossain, Stephen Bendall
}

\author{
Department of Trauma and Orthopaedics, Brighton and Sussex University Hospitals NHS Trust, Brighton, United Kingdom
}

Septic arthritis is a serious medical condition that can lead to significant complications if misdiagnosed or mismanaged. A rare case of a 1st metatarso-phalangeal joint septic arthritis in a native joint is presented in a patient with no significant risk factors. A 41-year-old patient was referred by his general practitioner owing to ongoing pain and swelling over his native 1st metatarso-phalangeal joint with difficulty on weightbearing for three months. After a series of investigations, including blood tests and a foot magnetic resonance imaging, which were inconclusive, the patient was led to the operating theatre for sampling and washout of his joint. The samples taken in the theatres revealed septic arthritis with Streptococcus mitis as the causative microorganism. The patient was treated with six weeks of oral antibiotics with a good functional outcome. This case report illuminates this rare condition and makes foot and ankle surgeons aware of its existence. A high suspicion for this condition can prevent misdiagnosis and mismanagement.

Key Words: Pseudopodagra, Infectious arthritis, Joints, Foot, Infection, Streptococcus mitis

Septic arthritis is an inflammatory condition caused by an infectious agent. It threatens the function of the joint by causing cartilage destruction rapidly. The incidence in developed countries is high (6 cases per 100,000 population each year) and the most vulnerable age groups are below 15 years and over 55 years. ${ }^{1)}$ The most commonly affected joints are the large joints of the body such as the hip and knee, foot and ankle joint infections occur in the $3 \%$ to $7 \%$ of the cases of septic arthritis. Important risk factors of the septic arthritis are the pre-existing joint pathologies such as the rheumatoid arthritis and the prosthetic joints. The causative microorganism migrates to the affected joint either directly from a wound (surgical or not) or from the bloodstream. In the later mechanism the source is another affected part of the body. Staphylococcus aureus is the most commonly isolated microorganism in septic arthritis and reaches the $40 \%$ of the cases in England and Wales. Other common causative microorganisms are the Streptococcus pneumoniae, Escherichia coli, and Proteus. ${ }^{1)}$

In the present study a rare case report of a 1st metatarso-pha-

Received January 21, 2019 Revised March 2, 2019 Accepted March 19, 2019 Corresponding Author: Efthymios Iliopoulos

Department of Trauma and Orthopaedics, Brighton and Sussex University Hospitals NHS Trust, Eastern Road, Brighton BN2 5BE, United Kingdom Tel: +447454202300, E-mail: iliopoulose@gmail.com

ORCID: https://orcid.org/0000-0003-3682-4303

Financial support: None.

Conflict of interest: None. langeal (MTP) joint infection by Streptococcus mitis is presented. The main key points of the rarity of this case are the unusual affected joint and the lack of risk factors. This case report has been approved by the Institutional Foot and Ankle Review Board of our hospital.

\section{CASE REPORT}

A 41-year-old patient was referred to our foot and ankle specialist service by his general practitioner (GP) due to ongoing pain and swelling over his 1st MTP joint, with difficulty on weight bear, without any history of trauma or foreign body. His symptoms started around 3 months prior to his first visit to our hospital. He was treated by his GP for possible gout (uric acid levels were at higher normal) and his symptoms improved but not completely resolved. The patient reports a discharge over his 1st MTP joint during this period, for which he received a two-week course of oral antibiotics by his GP. The discharge stopped and the swelling and pain was improved. The pain and swelling recurred when the antibiotics stopped, so he received one more course ( 7 days) of oral antibiotics by his GP.

His past medical history includes, acid reflux disease and hyperlipidaemia. He has a family history of premature coronary artery disease and he is an ex-smoker (stopped seven years ago) and ex- 
drinker (stopped five years ago). He has no history of diabetes or other metabolic disease.

On examination at the time of presentation his fore-foot was slightly swollen and red. There was no specific point of tenderness and there was no discharging sinuses or wounds around his foot. He had full range of motion of his 1st MTP joint, which was not painful. He had normal sensation and he had palpable dorsalis pedis and posterior tibialis pulses. He was finding it difficult to weight bear through the medial side of his forefoot. He had no history of trauma over his foot and he had no episodes of sepsis elsewhere recently.

Plain radiographs revealed mild osteopenia over the first metatarsal head and mild joint space narrowing of his 1st MTP joint (Fig. 1). His white blood cells (WBC) at the time of presentation were normal $\left(5.5 \times 10^{9} / \mathrm{L}\right.$; normal range, $\left.4 \sim 11 \times 10^{9} / \mathrm{L}\right)$ with normal neutrophils ( $46.4 \%, 2.6 \times 10^{9} / \mathrm{L}$; normal range, $2 \sim 7.5 \times 10^{9} / \mathrm{L}$ ). His erythrocyte sedimentation rate (ESR) was slightly elevated at $39 \mathrm{~mm} / \mathrm{hr}$ (normal range, $0 \sim 15 \mathrm{~mm} / \mathrm{hr}$ ) and his serum C-reactive protein $(\mathrm{CRP})$ at $7.6 \mathrm{mg} / \mathrm{L}$ (normal range, $0 \sim 5 \mathrm{mg} / \mathrm{L}$ ). Blood tests were similar with the ones performed a couple of months earlier by his GP, where the WBC were $8.1 \times 10^{9} / \mathrm{L}$, with ESR at $27 \mathrm{~mm} /$ $\mathrm{hr}$ and CRP at $8.5 \mathrm{mg} / \mathrm{L}$. Rheumatologic screening was normal.

As osteomyelitis was included to the differential diagnosis of the patient's condition he was send for an urgent magnetic resonance imaging (MRI) of his foot, which showed a small joint effusion and minor soft tissue swelling around the the 1st MTP joint. A mild marrow oedema at the plantar aspect of the 1st metatarsal head and the adjacent medial sesamoid was seen. Findings are suggesting to be reactive marrow changes rather than acute osteomyelitis (Fig. 2).

The patient was listed for biopsy of his 1st MTP joint to establish the diagnosis. An open biopsy was performed through a dorsal incision. Except from some small dorsal osteophytes, there were no other significant findings and the joint was macroscopically normal with no signs of arthritis. Joint fluid and tissue samples from the 1st MTP joint capsule were sent for histology and cultures.

The histology of the samples revealed a non-specific inflammatory arthropathy, with no signs of gout and the soft tissue samples grown $S$. mitis sensitive to penicillin and clarithromycin. The microscopy of the synovial fluid did not reveal any crystals. After discussing with our microbiologist colleagues a course of six weeks of oral clindamycin $450 \mathrm{mg}$ every 6 hours was started. The patient was given a short walker boot for mobilising.
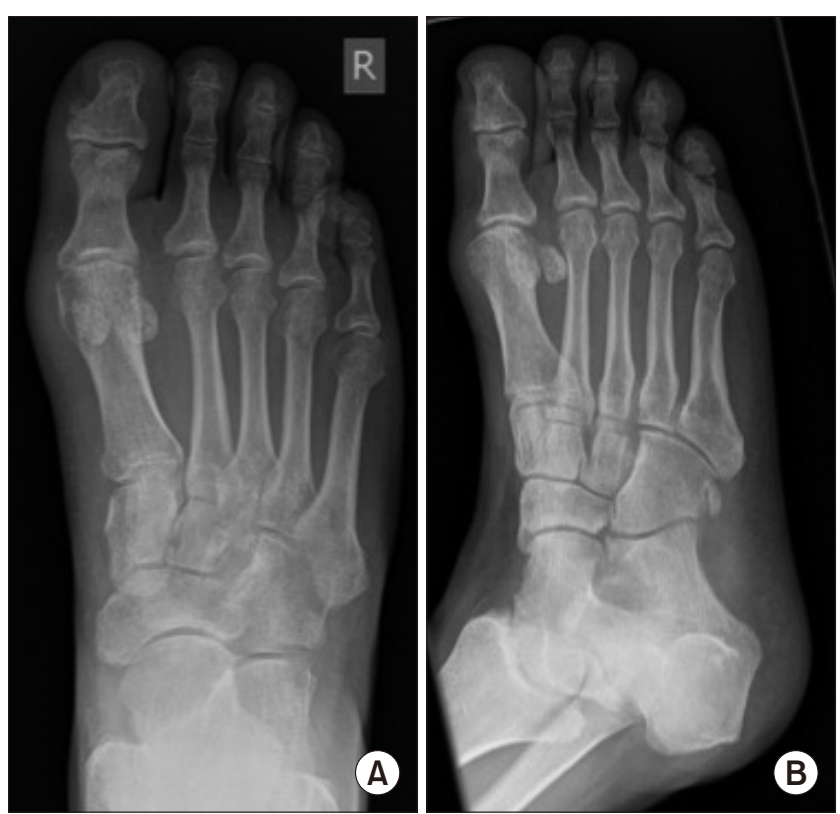

Figure 1. Anteroposterior (A) and oblique (B) plain radiographs at the time of presentation.
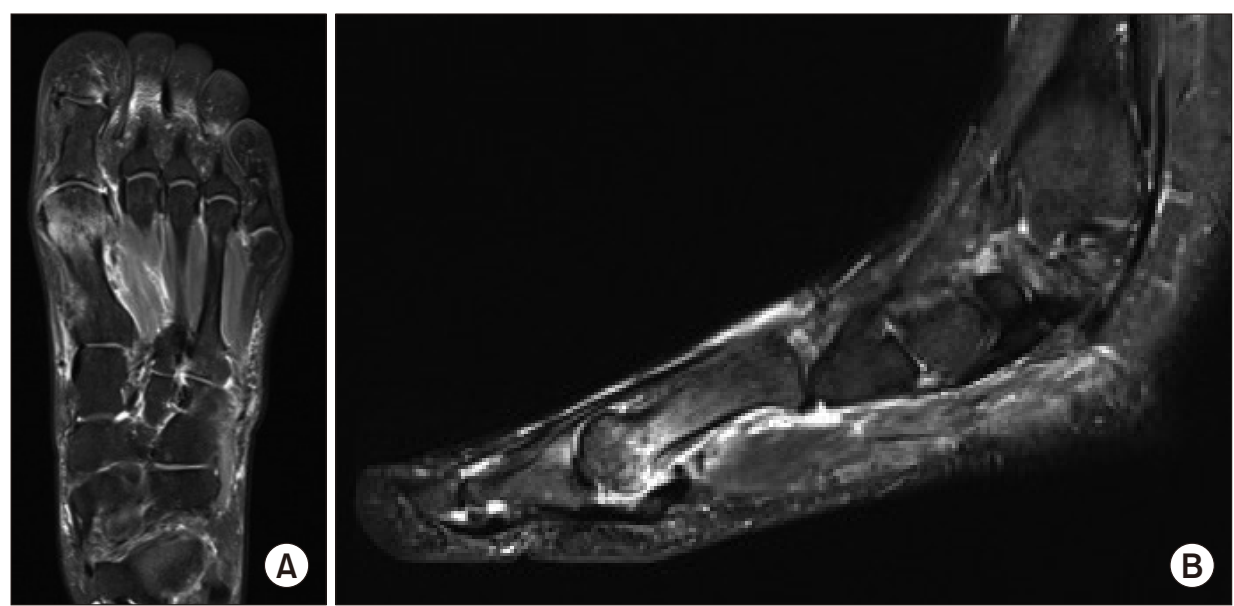

Figure 2. T2 sequence of the magnetic resonance imaging scans of the affected foot show the bone marrow oedema. (A) Axial view, (B) sagittal view. 
After the end of the antibiotic therapy the patient had no pain and swelling around his foot. His blood tests had returned to normal limits (WBC $5.6 \times 10^{9} / \mathrm{L}$, ESR $9 \mathrm{~mm} / \mathrm{hr}$, and CRP $5 \mathrm{mg} / \mathrm{L}$ ). Three months after the patient remained asymptomatic and had returned to normal activities with no pain or discomfort.

\section{DISCUSSION}

The present case report is the first to the authors' knowledge describing a septic arthritis of a native 1st metatarso-phalageal joint, without patient's risk factors. It illuminates that septic arthritis can be present to any joint, so the clinicians should have high suspicion for such pathology and add it to their differential diagnosis of a painful joint.

Pseudopodagra is a common clinical condition for which the GPs are asked to diagnose and treat accordingly. The differential diagnosis of a painful big toe includes except the most common cause of gout, a plethora of other clinical conditions, such as infection of the joint, the surrounding soft tissues or the bone (osteomyelitis), other rheumatoid conditions (ankylosing spondylitis, pyrophosphate arthropathy), arthritis and mechanical reasons. The diagnosis of gout has been traditionally use the urate crystals, leaving the serum urate levels aside, as it has been proven to be unreliable markers. In the present case report, the serum urate levels were at the highest normal, and the initial treatment for gout was unsuccessful, delaying the final diagnosis. This fact illuminates the importance of the consideration of the whole spectrum of differential diagnosis of pseudopodagra in the first place. Similar delays in the diagnosis of septic pseudopodagra have been reported previously in the literature by McClatchey and Goldman ${ }^{2)}$ and by Riera et al. ${ }^{3)}$

In foot and ankle the most commonly affected joint for infection is the tibio-talar joint and as in the rest of the body the most common causative microorganism is $S$. aureus. ESR and CPR, in contrast with WBC count, have been proven as sensitive markers for foot and ankle septic arthritis, findings which were present in the present case as well. Wynes et al. ${ }^{4)}$ report a patient with hypogammaglobulinemia who developed septic arthritis of the subtalar joint, caused by Mycoplasma hominis. This case was treated with incision and drainage and long term antibiotics with good final result. ${ }^{4)}$ Both McClatchey and Goldman ${ }^{2)}$ and Riera et al. ${ }^{3)}$ reported a 1st MTP joint septic arthritis caused by Hemophilus influenza and Streptococcus agalactiae respectively in immuno-compromised patients.
Overlying skin infection, older age (over 80 years), diabetes, prosthetic joint and rheumatoid arthritis, have been reported to be the most significant risk factors for septic arthritis in adults. ${ }^{5)}$ Immunosuppression, cancer and iatrogenic factors (i.e., intra-articular therapeutic injections) have been identified as risk factors as well. Pavic et al. () identified that crystal arthropathy is a risk factor for septic arthritis, supporting their finding to the abnormality of the joint due to the crystal arthropathy. The patient in the current case report had no history of gout. His GP has started him on medication for gout after his first presentation to him, based on his clinical presentation and not on the laboratory results. There were no evidence of gout in the samples obtained during the procedure, and the crystal arthropathy was excluded. On the MRI scan bone marrow oedema was identified, which did not have the appearance of osteomyelitis. Diabetes mellitus and intravenous drug abuse have been identified as risk factors for adjacent osteomyelitis of septic arthritis. These factors were not present in this case as well.

$S$. mitis is an uncommon cause of septic arthritis. It belongs to the streptococcus viridians group and is part of the common oral flora. Mitis is the most commonly isolated microorganism of the viridians group in bacteremia of immunocompromised patients after dental procedures. Due to that fact the American Dental Association and the American Academy of Orthopaedic Surgeons recommend antibiotic prophylaxis during dental procedures. Although periprosthetic infections after dental procedures are well documented in the hip and knee literature, there is only one report of foot and ankle peri-prosthetic infection. Young et al. ${ }^{7)}$ report a case of infected total ankle arthroplasty following a dental procedure, caused by $S$. mitis. The patient was treated with a two stage revision surgery alongside with long term antibiotics, which lead to good clinical outcome. ${ }^{7}$

Septic arthritis by $S$. mitis in non-replaced joints is even rarer. Yusuf et al. ${ }^{8)}$ reported a patient with septic arthritis of the pubic symphysis with retro-articular abscess formation caused by $S$. mitis. That patient was in an older age group and had similar clinical presentation with pain around the infected joint and no history of trauma of other source of sepsis. The CRP was elevated above 100 $\mathrm{mg} / \mathrm{L}$ at presentation and the rest of the blood tests were normal. Sequential treatment of intra-venous and oral antibiotics after the abscess aspiration led to cure. ${ }^{8)}$

Streptococci as cause of bone and joint infections are less common to staphylococci. Oral antibiotic therapy for six weeks with clindamycin $450 \mathrm{mg}$ four times a day was sufficient for the treatment of this infection. The selection of clindamycin as the antibi- 
otic therapy was made by our microbiology colleagues according to the cultures and sensitivity of the isolated $S$. mitis. Clindamicin is one of the most frequently used antibiotic for streptococci infections of bone and joint, and was proven effective in this case report. ${ }^{9)}$

Streptococci viridans and especially $S$. mitis have been reported to have the ability to colonise the heart valves and cause endocarditis. ${ }^{10)}$ Luckily our patient was treated adequately and on time without having such a significant complication. Targeted clinical examination and possible echocardiography should be considered when such microorganism is identified, with the view to identify early such a complication.

In conclusion, an acute 1st MTP joint infection in a native joint is reported. The lack of significant risk factors and the rarely affected joint makes this case quite rare. It is important for the clinicians to be suspicious for this condition when seeing patients with foot pain, in order to avoid delay on treatment or mismanagement.

\section{REFERENCES}

1. Nade S. Septic arthritis. Best Pract Res Clin Rheumatol. 2003;17:183-200. doi: 10.1016/S1521-6942(02)00106-7.

2. McClatchey WM, Goldman JA. Pseudopodagra from hemophilus influenza in an adult. Arthritis Rheum. 1979;22:681-3. doi: 10.1002/art.1780220607

3. Riera E, Mateo L, Sallés M, Pérez R. [Septic pseudopodagra by Streptococcus agalactiae]. Reumatol Clin. 2006;2:324-6. Spanish. doi: 10.1016/S1699-258X(06)73069-8.

4. Wynes J, Harris W 4th, Hadfield RA, Malay DS. Subtalar joint septic arthritis in a patient with hypogammaglobulinemia. J Foot Ankle Surg. 2013;52:242-8. doi: 10.1053/j.jfas.2012.10.012.

5. Kaandorp CJ, Dinant HJ, van de Laar MA, Moens HJ, Prins AP, Dijkmans BA. Incidence and sources of native and prosthetic joint infection: a community based prospective survey. Ann Rheum Dis. 1997;56:470-5. doi: 10.1136/ard.56.8.470.

6. Pavic K, Pandya J, Sebak S, Shetty A, Spencer D, Manolios N. Acute arthritis: predictive factors and current practice in the approach to diagnosis and management across two hospitals in Sydney. Intern Med J. 2018;48:1087-95. doi: 10.1111/imj.13969.

7. Young JL, May MM, Haddad SL. Infected total ankle arthroplasty following routine dental procedure. Foot Ankle Int. 2009;30:252-7. doi: 10.3113/FAI.2009.0252.

8. Yusuf E, Hofer M, Steinrücken J, Trampuz A, Borens O. Septic arthritis of the pubic symphysis caused by streptococcus mitis. Acta Clin Belg. 2014;69:454-5. doi: 10.1179/2295333714Y.0000 000059 .

9. Seng P, Vernier M, Gay A, Pinelli PO, Legré R, Stein A. Clinical features and outcome of bone and joint infections with streptococcal involvement: 5-year experience of interregional reference centres in the south of France. New Microbes New Infect. 2016;12:8-17. doi: 10.1016/j.nmni.2016.03.009.

10. Rapeport KB, Girón JA, Rosner F. Streptococcus mitis endocarditis. Report of 17 cases. Arch Intern Med. 1986;146:2361-3. doi: 10.1001/archinte.1986.00360240083014. 